\title{
ФИЗИКО-МАТЕМАТИЧЕСКИЕ НАУКИ
}

УДК 53.05

П. Ю. Гололобов, Г. С. Павлов

\section{Временной ход полусуточных вариаций интенсивности космических лучей по данным наземных детекторов за 1971-2021 гг.}

Институт космофизических исследований и аэрономии им. Ю.Г. Шафера СО РАН, г. Якутск, Россия

Аннотация. Регистрируемые наземными детекторами галактические космические лучи (КЛ) по пути своего распространения подвергаются воздействию основного воздействующего фактора - солнечного ветра, заполняющего всю гелиосферу. Модуляция космических лучей гелиосферой приводит к образованию их анизотропного пространственно-углового распределения в межпланетном пространстве. В данных наземных детекторов КЛ такая анизотропия проявляет себя вследствие вращения Земли вокруг своей оси в виде периодических суточных колебаний регистрируемой интенсивности. Параметры таких колебаний определяются состоянием солнечной активности (СА) и соответствующими изменениями крупномасштабной структуры. Как правило, амплитуда подобных колебаний мала и составляет лишь сотые доли от общего потока КЛ. Однако анизотропия КЛ служит важным источником информации о свойствах и структуре всей гелиосферы. Существующая на сегодня картина модуляции КЛ в гелиосфере предполагает существование их избытка с направлений поперек силовых линий межпланетного магнитного поля (ММП). Экспериментальные данные подтверждают такую картину, и в среднем максимум колебаний наблюдается как раз в 3 и 15 ч по местному времени. Учитывая, что направление силовых линий поля сохраняется почти неизменным, справедливо ожидать, что указанное выше время максимума должно оставаться также неизменным. Однако длительные наблюдения показали, что в зависимости от уровня СА время максимума может существенно смещаться

ГОЛОЛОБОВ Петр Юрьевич - м. н. с., Институт космофизических исследований и аэрономии им. Ю.Г. Шафера СО РАН.

E-mail: gpeter@ikfia.ysn.ru

GOLOLOBOV Petr Yurievich - Junior Researcher High energy cosmic ray laboratory Yu.G. Shafer Institute of Cosmophysical Research and Aeronomy of the Siberian Branch of the Russian Academy of Scinces.

ПАВЛОВ Гавриил Сергеевич - м. н. с. Лаборатории космических лучей высоких энергий, ИКФиА им. Ю.Г. Шафера СО РАН.

E-mail: ganya1981@mail.ru

PAVLOV Gavriil Sergeevich - Junior Researcher High energy cosmic ray laboratory Yu.G. Shafer Institute of Cosmophysical Research and Aeronomy of the Siberian Branch of the Russian Academy of Scinces. 
на раннее время. Данная работа представляет собой попытку объяснения наблюдаемых амплитудно-фазовых колебаний полусуточных вариаций интенсивности КЛ. На основе актуальных представлений о взаимодействии КЛ с атмосферой Земли и геомагнитным полем проведены расчеты ожидаемых полусуточных вариаций интенсивности КЛ, регистрируемых мировыми сетями мюонных детекторов и нейтронных мониторов. Сопоставление расчетных и наблюдательных данных показало, что наблюдаемое смещение возможно объяснить динамикой энергетического спектра полусуточных вариаций КЛ. Полученный результат подтверждает и дополняет полученные ранее другими авторами результаты, а также ее можно рассматривать как указание для дальнейшего развития теории гелиосферной модуляции КЛ.

Ключевые слова: космические лучи, полусуточные вариации, анизотропия КЛ, энергетический спектр, межпланетное магнитное поле, мюонный телескоп, нейтронный монитор, солнечная активность, гелиосфера, вторичные КЛ, атмосфера.

Работа в рамках проекта Министерства науки и высшего образования Российской Федерации №AAАA-А21-121011890014-0 с использованием оборудования уникальной научной установки «Российская национальная наземная сеть станций космических лучей». Авторы благодарны иентру данных по космической физике NASA/GSFC (https://omniweb.gsfc.nasa.gov/), базам данных WDCCR (https://cidas.isee.nagoya-u.ac.jp/WDCCR/) университета Нагоя, Япония и GMDN (http:// cosray.shinshu-u.ac.jp/crest) университета Синсю, Япония за предоставленные данные.

DOI

\author{
P. Yu. Gololobov, G. S. Pavlov
}

\title{
Time course of semidiurnal variations in the intensity of cosmic rays according to the data of ground-based detectors for 1971-2021.
}

\author{
Federal State Budgetary Scientific Institution Yu.G. Shafer Institute of Cosmophysical Research \\ and Aeronomy of the Siberian Branch of the Russian Academy of Sciences, Yakutsk, Russia
}

Abstract. Galactic cosmic rays (CR) registered by ground-based detectors are subjected to the main influencing factor along their propagation path - the solar wind, which fills the entire heliosphere. Modulation of $\mathrm{CR}$ by the heliosphere leads to the formation of their anisotropic spatial-angular distribution in interplanetary space. In the data of ground-based detectors, such anisotropy manifests itself, due to the rotation of the Earth around its axis, in the form of periodic daily fluctuations in the registered intensity. The parameters of such fluctuations are determined by the state of solar activity and the corresponding changes in the large-scale structure. As a rule, the amplitude of such oscillations is small and amounts to only hundredths of the total flux of CR. However, the anisotropy of $\mathrm{CR}$ is an important source of information about the properties and structure of the entire heliosphere. The current picture of modulation of $\mathrm{CR}$ in the heliosphere suggests the existence of their excess from directions across the lines of force of the interplanetary magnetic field. Experimental data confirm this picture and, on average, the maximum of fluctuations is observed within 3 and 15 hours local time. Considering that the direction of the field lines remains almost unchanged, it is fair to expect that the aforementioned maximum time should also remain unchanged. However, long-term observations have shown that, depending on the level of solar activity, the time of the maximum can significantly shift to an earlier time. This paper is an attempt to explain the observed amplitude-phase oscillations of semidiurnal variations in the intensity of cosmic rays. Based on current ideas about the interaction of CR with the Earth's atmosphere and the geomagnetic field, calculations of the expected semidiurnal variations in the intensity of CR recorded by world networks of muon detectors and neutron monitors have been carried out. Comparison of the calculated and observational data has shown that the observed shift can be explained by the dynamics of the energy spectrum of semidiurnal CR variations. The result obtained confirms and 
supplements the results obtained earlier by other authors, and it can also be considered as an indication for the further development of the theory of heliospheric CR modulation.

Keywords: cosmic rays, semidiurnal variations, energy spectrum, CR anisotropy, interplanetary magnetic field, muon telescope, neutron monitor, solar activity, heliosphere, secondary CR, atmosphere.

The study was done under the project of the Ministry of Science and Higher Education of Russian Federation №AAAA-A21-121011890014-0 with the use of the Unique Research Facility "Russian National Ground Network of Cosmic Ray Stations". Authors express gratitude to the Space Physics Data Facility of NASA at the Goddard Space Flight Center, USA (https://omniweb.gsfc.nasa.gov), WDCCR database at Nagoya University, Japan (https://cidas.isee.nagoya-u.ac.jp/WDCCR/), and the Global Muon Detection Network station at Shinshu University, Japan (http://cosray.shinshu-u.ac.jp/crest) for the provided data.

\section{Введение}

Гелиосферная модуляция КЛ имеет анизотропный характер и вследствие вращения Земли вокруг своей оси регистрируется наземными детекторами в виде периодических суточных колебаний. Существование второго углового момента этой анизотропии подтверждается наличием 12-часовой гармоники суточных вариаций интенсивности КЛ, регистрируемых наземными детекторами. Как правило, амплитуды таких колебаний малы и составляют порядка $0,1 \%$ и фазу около 3 и 15 ч по местному времени [1].

С обнаружения этого явления во второй половине прошлого столетия проводились попытки его теоретического осмысления, которые привели к возникновению нескольких успешных гипотез. В частности, эти теории представлены в ряде работ [2-8]. Не вдаваясь в детали, отметим, что физические механизмы, предлагаемые в этих работах, исходили из представлений о распространении КЛ в гелиосфере и объясняли избыток частиц поперек силовых линий ММП. При этом очевидно, что фаза вариаций должна была оставаться практически неизменной, так как закрутка силовых линий ММП слабо меняется со временем. Действительно, фаза полусуточных вариаций КЛ в основном не испытывает существенных изменений с циклами солнечной активности. Однако, как было обнаружено в ходе мониторинга состояния углового распределения КЛ на сайте https://www.ysn.ru/ starodub/SpaceWeather/global_survey_real_time.html, фаза полусуточных вариаций КЛ преимущественно была направлена на раннее время, около 0-1 ч по местному времени. Действительно, обработка длительного ряда экспериментальных данных показывает, что подобное смещение фазы наблюдается в периоды минимумов СА в эпоху положительной полярности ОМПС (рис. 1). Такое явление выходит за рамки существующих теорий образования полусуточных вариаций КЛ. Данная работа представляет собой попытку объяснения наблюдаемых амплитуднофазовых колебаний полусуточных вариаций интенсивности КЛ.

\section{Материалы и методы исследования}

Традиционные детекторы КЛ (нейтронные мониторы и мюонные телескопы) регистрируют т. н. «вторичные КЛ» - продукты взаимодействия КЛ с веществом атмосферы. Количество сгенерированных вторичных частиц (множественность) определяется энергией первичной частицы и растет с ее ростом. С другой стороны, поток КЛ в межпланетном пространстве уменьшается с ростом энергии. Совокупность этих факторов определяет чувствительность детектора к энергии КЛ, которая отражена в понятии коэффициентов связи, впервые предложенном Дорманом [9]. На сегодняшний 


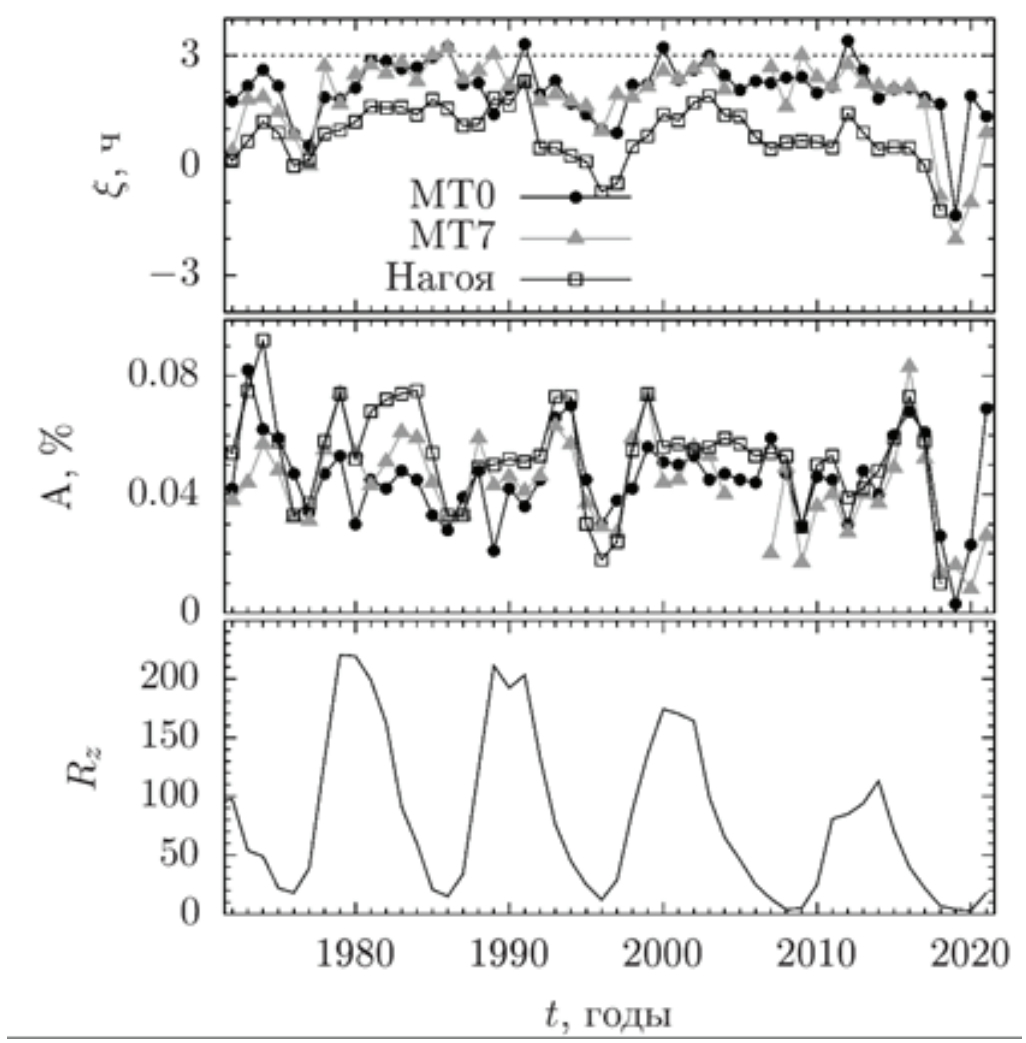

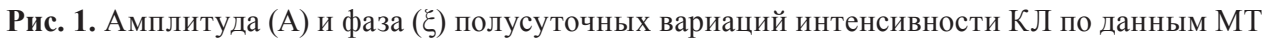

Якутского спектрографа КЛ на уровнях 0 и 7 м в.э. и МТ станции Нагоя за 1972-2021 гг.

В нижней панели представлено число солнечных пятен Вульфа (Rz) согласно базе данных OMNI [1]

день коэффициенты связи для большинства наземных детекторов КЛ можно считать известными. $\mathrm{C}$ исследованиями по определению коэффициентов связи можно ознакомиться, например, в работах [10-14].

Являясь заряженными частицами, КЛ испытывают воздействие геомагнитного поля. В основном это приводит к искривлению траекторий их движения и сносу полем. Как правило, величина сноса может быть отражена в т. н. асимптотических углах прихода частиц, связывающих направления прихода частиц к поверхности Земли и их точку входа в геомагнитное поле. Кроме того, геомагнитное поле также выполняет роль экранирования КЛ в результате возникновения в ней запрещенных траекторий. В результате возникают т. н. геомагнитные жесткости обрезания Rc, устанавливающие нижний предел жесткости заряженных частиц для достижения поверхности Земли. В грубом приближении можно сказать, что значение Rc увеличивается в направлении от полюсов к экватору Земли.

Расчет траекторий КЛ в геомагнитном поле производится путем численного интегрирования уравнений движения заряженных частиц в магнитных полях, описанного в [15], а также в [16]. Для задачи геомагнитного поля нами было использовано Международное геомагнитное аналитическое поле IGRF (https://www.ngdc.noaa.gov/IAGA/ vmod/igrf.html).

Взаимодействие КЛ с атмосферой Земли и геомагнитным полем приводит к тому, что вариации ее интенсивности, наблюдаемые наземными детекторами, отличаются от тех, которые наблюдаются в межпланетной среде. Для учета этого эффекта мы будем основываться на идее приемных векторов, предложенных якутскими космофизиками 
в 1960-х гг. [17, 18]. В основе метода лежат указанные выше представления о взаимодействии КЛ с геомагнитным полем и атмосферой Земли. При этом также учитываются и индивидуальные особенности геометрии детектора, определяемые диаграммами направленности $N(\theta, \varphi)$. В общем виде выражение для определения приемных векторов имеет вид:

$$
z_{n}^{m}=\frac{\int_{E_{m i n}}^{\infty} \int_{0}^{2 \pi} \int_{0}^{\pi / 2} W(E, \theta) f_{n}(E) N(\theta, \varphi) \sin \theta e^{i m^{\prime \prime}(E, \theta, \varphi)} P_{n}^{m}\left[\sin _{\uparrow}(E, \theta, \varphi)\right] d E d \theta d \varphi}{\int_{E_{\min }}^{\infty} \int_{0}^{2 \pi} \int_{0}^{\pi / 2} W(E, \theta) N(\theta, \varphi) \sin \theta d E d \theta d \varphi},
$$

где $E, \theta, \varphi-$ энергия, географическая долгота и широта, $W(E, \theta)-$ коэффициенты связи, $\Phi, \Psi$ - асимптотические углы прихода частиц, $f_{\mathrm{n}}(\mathrm{E})$ - энергетический спектр вариаций.

В итоге амплитуды и фазы первичных $(A, \xi)$ и, соответственно, ожидаемых вторичных $\left(A_{i}^{\prime}, \xi_{i}{ }^{\prime}\right)$ вариаций, наблюдаемых на $i$-м детекторе КЛ, будут связаны выражениями $A_{i}^{\prime}=A \sqrt{\chi_{n, i}^{m 2}+y_{n, i}^{m 2}}$ и $\xi-\xi_{i}^{\prime}=\arctan y_{n, i}^{m} / x_{n, i}^{m}$, соответственно.

Единственным параметром в выражении (1), свободным и неизвестным для нас, остается $f_{n}(E)$. Игнорируя различные теоретические предполагаемые виды спектров, будем грубо предполагать его плоским с верхним $E_{1}$ и нижним $E_{0}$ порогами обрезания: $f(E)=1$ при $E_{0} \leq E \leq E_{l}, f(E)=1$ при $E<E_{0}$ и $E>E_{l}$.

Теперь, подбирая значения $E_{l}$ и $E_{0}$, можно с помощью метода наименьших квадратов добиться наилучшего согласия рассчитанных данных $A_{i}{ }^{\prime}$ и $\xi_{i}{ }^{\prime}$ с реальными наблюдаемыми данными. При этом будем ограничивать фазу первичных вариаций $\xi$ в пределах $1,5-4,5$ ч по местному времени, опираясь на результаты исследований Смита и Бибера [19] об изменениях закрутки силовых линий ММП с циклами солнечной активности. Это позволит объяснить вариации фазы полусуточных вариаций КЛ, не противореча существующим теориям.

Для охвата наибольшего энергетического диапазона привлечем к анализу данные низкоширотных и высокоширотных нейтронных мониторов, а также мюонные телескопы, расположенные на разных уровнях под Землей. Список использованных детекторов КЛ представлен в табл.

Таблица

\begin{tabular}{|c|c|c|c|c|}
\hline № & Станция & $\begin{array}{c}\text { Географические } \\
\text { координаты }\end{array}$ & $\begin{array}{c}\text { высота над } \\
\text { уровнем моря, } \\
\text { м / глубина, } \\
\text { м в.э. }\end{array}$ & $\begin{array}{c}\text { Жесткость } \\
\text { геомагнитного } \\
\text { обрезания, ГВ }\end{array}$ \\
\hline \multicolumn{5}{|c|}{ Нейтронные мониторы } \\
\hline 1 & Апатит & $67.55 \mathrm{~N} 33.33 \mathrm{E}$ & $177 / 0$ & 0.57 \\
\hline 2 & Клаймакс & $39.37 \mathrm{~N} 106.18 \mathrm{~W}$ & $3400 / 0$ & 2.99 \\
\hline 3 & Ломницкий штит & $49.20 \mathrm{~N} 20.22 \mathrm{E}$ & $2634 / 0$ & 3.98 \\
\hline 4 & Якутск & $62.01 \mathrm{~N} 129.43 \mathrm{E}$ & $105 / 0$ & 1.65 \\
\hline 5 & Туле & $76.50 \mathrm{~N} 68.7 \mathrm{~W}$ & $26 / 0$ & 0.00 \\
\hline 6 & Рим & $41.90 \mathrm{~N} 12.52 \mathrm{E}$ & $60 / 0$ & 6.32 \\
\hline 7 & Халеакала & $20.72 \mathrm{~N} 156.27 \mathrm{~W}$ & $3030 / 0$ & 12.91 \\
\hline \multicolumn{5}{|c|}{ Мюонные телескопы } \\
\hline 8 & Нагоя & $35.15 \mathrm{~N} 136.97 \mathrm{E}$ & $77 / 0$ & 11.50 \\
\hline 9 & \multirow{3}{*}{ Якутск } & \multirow{3}{*}{$62.01 \mathrm{~N} 129.43 \mathrm{E}$} & $105 / 0$ & \multirow{3}{*}{01.65} \\
\hline 10 & & & $105 / 7$ & \\
\hline 11 & & & $105 / 20$ & \\
\hline 12 & Мисато & $36.12 \mathrm{~N} 137.50 \mathrm{E}$ & $735 / 30$ & 11.39 \\
\hline 13 & Сакашита & $35.58 \mathrm{~N} 137.53 \mathrm{E}$ & $334 / 80$ & 11.50 \\
\hline 14 & Матсуширо & $35.15 \mathrm{~N} 136.97 \mathrm{E}$ & $408 / 220$ & 11.50 \\
\hline
\end{tabular}



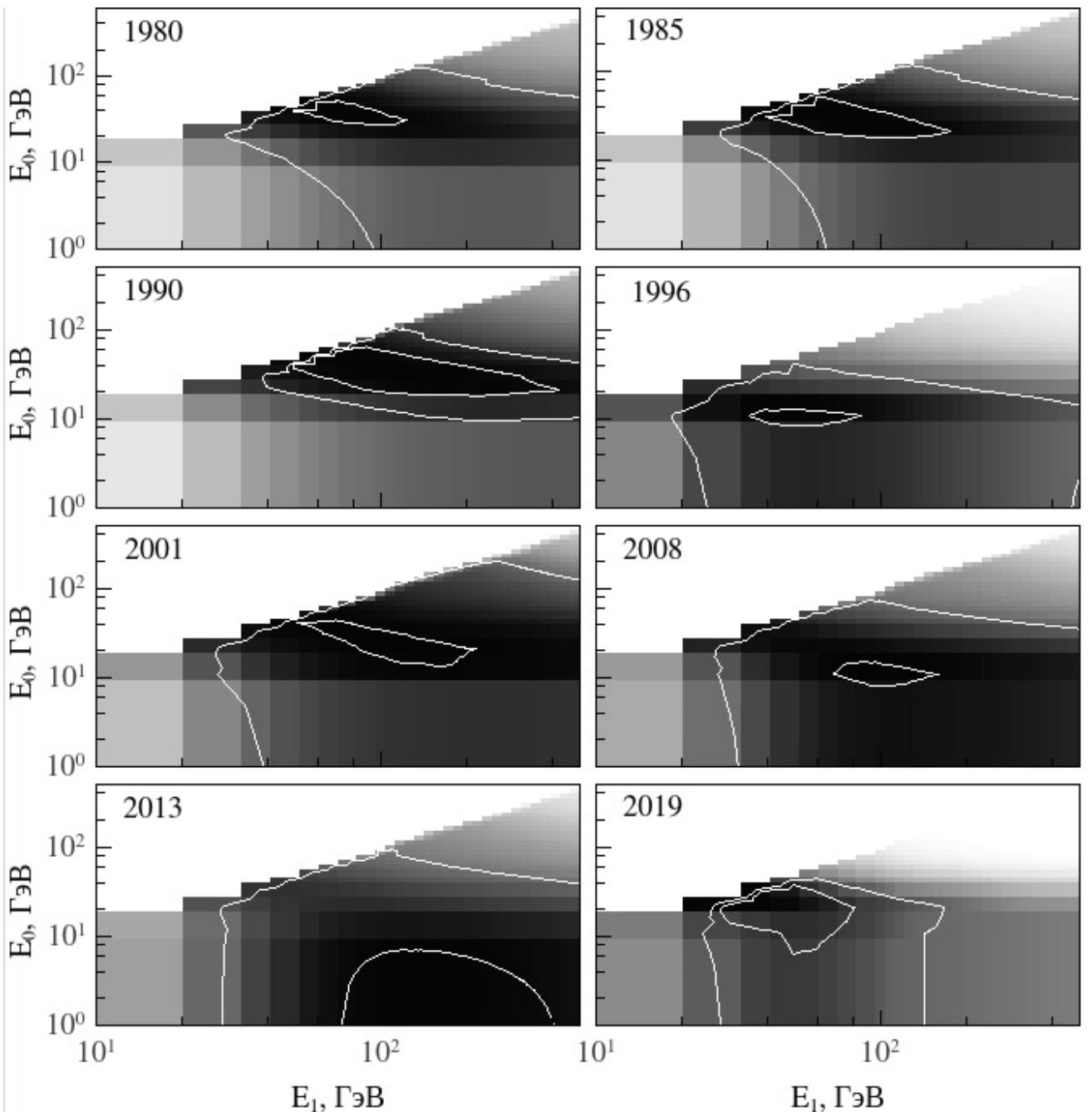

$10^{1}$

$10^{2}$

$\mathrm{E}_{1}$, ГэВ

Рис. 2. Контурная карта минимальных отклонений расчетных и наблюдательных данных при различных значения нижнего $E_{0}$ и верхнего $E_{1}$ порогов обрезания энергетического спектра полусуточных вариаций КЛ для периодов максимумов (1980, 1990, 2001, 2013 гг.) и минимумов $(1985,1996,2008,2019$ гг.) солнечной активности. Переход оттенка от светлого к темному соответствует уменьшению минимальных отклонений

\section{Результаты и обсуждение}

Результаты сопоставления теоретически ожидаемых и наблюдательных значений параметров полусуточной анизотропии КЛ для различных значений $E_{l}$ и $E_{0}$ представлены на рис. 2 в виде контурных карт распределений минимальных отклонений между экспериментальными и модельными расчетами. Отдельно рассматривались периоды минимумов и максимумов СА.

Как следует из рисунка, в периоды максимумов солнечной активности спектр должен быть жестким и ограничен 30-100 ГэВ. Немного мягким спектр наблюдается и в периоды минимумов солнечной активности в qA < 0 (1984-1986 гг., 2007-2009 гг.). В периоды минимумов СА при qA >0 наблюдается резкое смягчение спектра, при котором спектр оказывается ограниченным сверху $~ 50$ ГэВ. При этом отметим, что последний минимум СА характеризовался наиболее мягким спектром. По-видимому, это является следствием тенденции ослабления СА и соответствующего ослабления модуляции КЛ. 


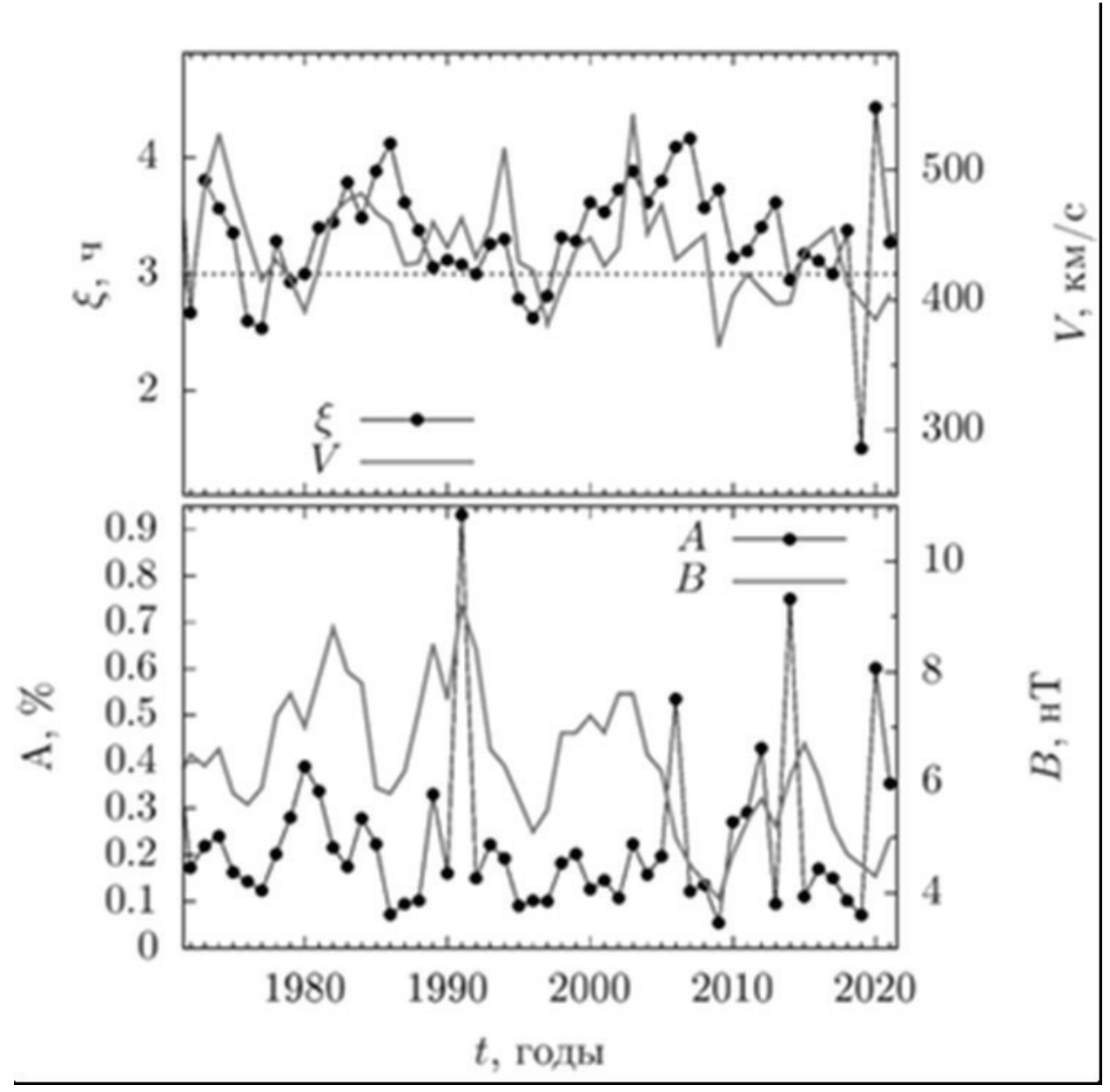

Рис. 3. Значение амплитуды и фазы полусуточной вариации КЛ $A$, $\xi$, соответствующее наилучшему согласию модельных расчетов и экспериментальных данных

(кривые с точками). Также представлены значения скорости солнечного ветра $V$ и модуля вектора ММП $B$ согласно базе данных OMNI

Рассмотрим временной ход среднегодовых значений амплитуды $A$ и фазы $\xi$ полусуточной вариации КЛ, которые соответствуют наилучшему согласию модельных расчетов и наблюдательных данных, за 1971-2021 гг. Полученные значения $A$ и $\xi$, а также скорость солнечного ветра и напряженность ММП представлены на рис. 3. Видно, что амплитуда $A$ положительно коррелирует с напряженностью ММП, что может быть связано с изменением уровня модуляции КЛ. Из рисунка также следует, что фаза $\xi$ также испытывает незначительные вариации, которые согласуются со скоростью солнечного ветра. Это связано с тем, что повышение скорости солнечного ветра сопровождается выпрямлением силовых линий ММП, и, наоборот, ее понижение закруткой.

Исходя из вышеуказанного, можно сделать вывод, что наблюдаемое смещение фазы полусуточных вариаций КЛ на раннее время может удовлетворительно объясняться изменениями ее энергетического спектра. Тем не менее следует признать, что использованный нами энергетический спектр второй сферической гармоники является грубым и может сильно отличаться от действительности. В работе Крымского и др. [20] предполагалось, что наблюдаемые полусуточные вариации КЛ обусловлены 
экранирующим воздействием регулярного ММП, создающего избыток частиц с направления поперек поля. При этом энергетический спектр, следующий из этой теории, имеет вид: $E_{1}^{\gamma}{ }_{1}$ при $E<E_{\max }$ и $E{ }_{2}{ }_{2}$ при $E<E_{\max }$, где $\gamma_{1}=1$ и $\gamma_{2}=-2$, а характерная энергия $E_{0}$ определялась параметрами ММП. Анализ экспериментальных данных позволил оценить $E_{\max }$ в 70 ГэВ. Подобный спектр и значения $E_{\max }$ были также получены Зусмановичем и Миркиным в работе [21]. С другой стороны, по оценкам Алувальи и Фикани [22], показатели энергетического спектра составляли несколько другие значения $\gamma_{1}=0,7 \pm 0,3$ и $\gamma_{2}=0,4 \pm 0,2$, а $E_{\max }$ зависела от цикла СА, достигая 50 ГэВ в минимуме солнечной активности и 150 ГэВ в максимуме. Несмотря на несколько различные оценки энергетического спектра, полученные в вышеуказанных работах, в целом можно утверждать, что полусуточные вариации интенсивности КЛ должны наблюдаться в области энергий, близких к 70 ГэВ, и варьироваться в пределах 50-150 ГэВ в зависимости от цикла солнечной активности. Поэтому можно заключить, что полученные в данной работе выводы о динамике энергетического спектра полусуточной вариации КЛ с циклами СА согласуются с работами других авторов.

\section{Заключение}

Проведен анализ поведения полусуточных вариаций интенсивности КЛ, наблюдаемых наземными детекторами, за период с 1971 по 2021 гг. Показано, что наблюдаемые необычные смещения фазы вариаций на раннее время не согласуется с теоретическими представлениями. Установлено, что подобное может удовлетворительно объясняться изменениями ее энергетического спектра полусуточных вариаций КЛ. Наиболее мягкий спектр наблюдается в периоды минимумов солнечной активности при положительной полярности ОМПС. Полученный результат можно рассматривать как указание для дальнейшего развития теории гелиосферной модуляции КЛ.

\section{Л и т е р а т у р а}

1. King, J. H. Solar wind spatial scales in and comparisons of hourly Wind and ACE plasma and magnetic field data / J. H. King, N. E. Papitashvili // Journal of Geophysical Research. - 2005. - V. 110. - № A02104. - P. 1-8.

2. Subramanian, G. Consequences of the distribution of galactic cosmic-ray density in the solar system / G. Subramanian, V. Sarabhai // The Astrophysical Journal. - 1967. - V. 149. - P. 417-428. doi: 10.1086/149266.

3. Quenby, J. J. The second harmonic of the cosmic ray daily variation / J. J. Quenby, B. Lietti // Planetary and Space Science. - 1968. - V. 16. - P. 1209-1219. doi: 10.1016/0032-0633(68)90027-5.

4. Sarabhai, V. Anisotropy of galactic cosmic rays and the interplanetary magnetic field / V. Sarabhai, G. L. Pai, M. Wada // Nature. - 1965. - V. 206. - P. 703-704. doi: 10.1038/206703a0.

5. Sarabhai, V. Galactic cosmic rays in the solar system / V. Sarabhai, G. Subramanian // The Astrophysical Journal. - 1966. - V. 145. - P. 206-214.

6. Nagashima, K. Three-dimensional cosmic ray anisotropy in interplanetary space. Part III — Origin of Cosmic Ray Solar Semidiurnal Variation / K. Nagashima, H. Ueno, K. Fujimoto [et al] // Report of ionosphere and space research in Japan. - 1972. - V. 26. - P. 1-30.

7. Nagashima, K. Three-dimensional cosmic ray anisotropy in interplanetary space. Part IV - Origin of Solar Semidiurnal Variation / K. Nagashima, K. Fujimoto, Z. Fujii [et al.] // Report of ionosphere and space research in Japan. - 1972. - V. 26. - P. 31-68.

8. Krivoshapkin, P. A. The second spherical harmonics in the distribution of cosmic rays / P. A. Krivoshapkin, G. F. Krymsky, A. I. Kuzmin [et al.] // Acta Physica Academiae Scientiarum Hungaricae. - 1970. - V. 29. - P. 147-151.

9. Дорман, Л. И. Вариации космических лучей: монография / Л. И. Дорман. - Москва : Гостехиздат. $-1957 .-492$ C.

10. Nuntiyakul, W. Latitude survey investigation of galactic cosmic ray solar modulation during 1994-2007 / W. Nuntiyakul, P. Evenson, D. Ruffolo [et al.] // The Astrophysical Journal. - 2014. - V. 795:11. - Issue 5. -13 pp. doi:10.1088/0004-637X/795/1/11. 
11. Крымский, Г. Ф. Модель генерации мюонов в земной атмосфере / Г. Ф. Крымский, П. А. Кривошапкин, В. Г. Григорьев // Геомагнетизм и аэрономия. 2011. - Т. 51. - №5. - С. 716-720.

12. Алексаньян, Т. М. Экспериментальные исследования геомагнитных эффектов в космических лучах и спектр эффекта возрастания перед магнитными бурями / Т. М. Алексаньян, И. В. Дорман, Л. И. Дорман [др.] // Известия АН СССР. Серия физическая, журнал. - 1982. - Т. 46. - №9. С. 1689-1691.

13. Fujimoto, K. Coupling coefficients of the cosmic ray variations for meson telescopes / K. Fujimoto, S. Yasue, I. Kondo, K. Nagashima // Proceedings of 15th International Cosmic Ray Conference. Budapest, Bulgaria. - 1977. - V. 4. - P. 321-325.

14. Murakami, K. Response functions for cosmic-ray muons at various depths underground / K. Murakami, K. Nagashima, S. Sagisaka [et al.] // Il Nuovo Cimento. - 1979. - V. 2 C. - No. 5. - P. 635-651.

15. Дорман, Л. И. Космические лучи в магнитном поле Земли : монография / Л. И. Дорман В. С. Смирнов, М. И. Тясто. - Москва : Наука. - 1971. - 400 С.

16. Smart, D. F. Magnetospheric models and trajectory computations / D. F. Smart, M. A. Shea, E. O. Flückiger // Space Science Reviews. - 2000. - V. 93. - P. 305-333. doi: 10.1023/A:1026556831199.

17. Крымский, Г. Ф. Распределение космических лучей и приемные векторы детекторов. I / Г. Ф. Крымский, А. И. Кузьмин, Н. П. Чирков [и др.] // Геомагнетизм и аэрономия. - 1966. - Т. 6. - № 3. - С. 991-996.

18. Крымский, Г. Ф. Распределение космических лучей и приемные векторы детекторов. II / А. И. Кузьмин, Н. П. Чирков, П. А. Кривошапкин [и др.] // Геомагнетизм и аэрономия. - 1967. - Т. 7. - № 1. - C. 11-15.

19. Smith, C. W. Solar cycle variations of the interplanetary magnetic field spiral / C. W. Smith, J. W. Bieber // The Astrophysical Journal. - 1991. - V. 370. - P. 435-441.

20. Космические лучи и солнечный ветер: монография / Г. Ф. Крымский А. И. Кузьмин, П. А. Кривошапкин [и др.] - Новосибирск : Наука. - 1981. - 224 с.

21. Zusmanovich, A. G. Energy Spectrum of the Semidiurnal Variation of Cosmic Rays / A. G. Zusmanovich, L. A. Mirkin // Proc. 18Th ICRC. - 1983. - P. 333-336.

22. Ahluwalia, H. S. Cosmic ray solar semidiurnal anisotropy. 1. Treatment of experimental data / H. S. Ahluwalia, M. M. Fikani // Journal of Geophysical Research. - 1996. - V. 101. - No. A5. P. 11075-11085.

\section{Ref e r e n e s}

1. King, J. H. Solar wind spatial scales in and comparisons of hourly Wind and ACE plasma and magnetic field data / J. H. King, N. E. Papitashvili // Journal of Geophysical Research. - 2005. - V. 110. - № A02104. - P. 1-8.

2. Subramanian, G. Consequences of the distribution of galactic cosmic-ray density in the solar system / G. Subramanian, V. Sarabhai // The Astrophysical Journal. - 1967. - V. 149. - P. 417-428. doi: 10.1086/149266.

3. Quenby, J. J. The second harmonic of the cosmic ray daily variation / J. J. Quenby, B. Lietti // Planetary and Space Science. - 1968. - V. 16. - P. 1209-1219. doi: 10.1016/0032-0633(68)90027-5.

4. Sarabhai, V. Anisotropy of galactic cosmic rays and the interplanetary magnetic field / V. Sarabhai, G. L. Pai, M. Wada // Nature. - 1965. - V. 206. - P. 703-704. doi: 10.1038/206703a0.

5. Sarabhai, V. Galactic cosmic rays in the solar system / V. Sarabhai, G. Subramanian // The Astrophysical Journal. - 1966. - V. 145. - P. 206-214.

6. Nagashima, K. Three-dimensional cosmic ray anisotropy in interplanetary space. Part III - Origin of Cosmic Ray Solar Semidiurnal Variation / K. Nagashima, H. Ueno, K. Fujimoto [et al] // Report of ionosphere and space research in Japan. - 1972. - V. 26. - P. 1-30.

7. Nagashima, K. Three-dimensional cosmic ray anisotropy in interplanetary space. Part IV - Origin of Solar Semidiurnal Variation / K. Nagashima, K. Fujimoto, Z. Fujii [et al.] // Report of ionosphere and space research in Japan. - 1972. - V. 26. - P. 31-68. 
8. Krivoshapkin, P. A. The second spherical harmonics in the distribution of cosmic rays / P. A. Krivoshapkin, G. F. Krymsky, A. I. Kuzmin [et al.] // Acta Physica Academiae Scientiarum Hungaricae. - 1970. - V. 29. - P. 147-151.

9. Dorman, L. I. Variacii kosmicheskih luchej: monografiya / L. I. Dorman. - Moskva : Gostekhizdat. 1957. $-492 \mathrm{~S}$.

10. Nuntiyakul, W. Latitude survey investigation of galactic cosmic ray solar modulation during 1994-2007 / W. Nuntiyakul, P. Evenson, D. Ruffolo [et al.] // The Astrophysical Journal. - 2014. - V. 795:11. - Issue 5. - 13 pp. doi:10.1088/0004-637X/795/1/11.

11. Krymskij, G. F. Model' generacii myuonov v zemnoj atmosfere / G. F. Krymskij, P. A. Krivoshapkin, V. G. Grigor'ev // Geomagnetizm i aeronomiya. 2011. - T. 51. - №5. - S. 716-720.

12. Aleksan'yan, T. M. Eksperimental'nye issledovaniya geomagnitnyh effektov v kosmicheskih luchah i spektr effekta vozrastaniya pered magnitnymi buryami / T. M. Aleksan'yan, I. V. Dorman, L. I. Dorman [dr.] // Izvestiya AN SSSR. Seriya fizicheskaya, zhurnal. - 1982. - T. 46. - №9. - S. 1689-1691.

13. Fujimoto, K. Coupling coefficients of the cosmic ray variations for meson telescopes / K. Fujimoto, S. Yasue, I. Kondo, K. Nagashima // Proceedings of 15th International Cosmic Ray Conference. Budapest, Bulgaria. - 1977. - V. 4. - P. 321-325.

14. Murakami, K. Response functions for cosmic-ray muons at various depths underground / K. Murakami, K. Nagashima, S. Sagisaka [et al.] // Il Nuovo Cimento. - 1979. - V. 2 C. - No. 5. - P. 635-651.

15. Dorman, L. I. Kosmicheskie luchi v magnitnom pole Zemli : monografiya / L. I. Dorman V. S. Smirnov, M. I. Tyasto. - Moskva : Nauka. - 1971. - 400 S.

16. Smart, D. F. Magnetospheric models and trajectory computations / D. F. Smart, M. A. Shea, E. O. Flückiger // Space Science Reviews. - 2000. - V. 93. - P. 305-333. doi: 10.1023/A:1026556831199.

17. Krymskij, G. F. Raspredelenie kosmicheskih luchej i priemnye vektory detektorov. I / G. F. Krymskij, A. I. Kuz'min, N. P. Chirkov [i dr.] // Geomagnetizm i aeronomiya. - 1966. - T. 6. - № 3. - S. 991-996.

18. Krymskij, G. F. Raspredelenie kosmicheskih luchej i priemnye vektory detektorov. II / A. I. Kuz'min, N. P. CHirkov, P. A. Krivoshapkin [i dr.] // Geomagnetizm i aeronomiya. - 1967. - T. 7. - № 1. - S. 11-15.

19. Smith, C. W. Solar cycle variations of the interplanetary magnetic field spiral / C. W. Smith, J. W. Bieber // The Astrophysical Journal. - 1991. - V. 370. - P. 435-441.

20. Kosmicheskie luchi i solnechnyj veter: monografiya / G. F. Krymskij A. I. Kuz'min, P. A. Krivoshapkin [i dr.] - Novosibirsk : Nauka. - 1981. - 224 s.

21. Zusmanovich, A. G. Energy Spectrum of the Semidiurnal Variation of Cosmic Rays / A. G. Zusmanovich, L. A. Mirkin // Proc. 18Th ICRC. - 1983. - P. 333-336.

22. Ahluwalia, H. S. Cosmic ray solar semidiurnal anisotropy. 1. Treatment of experimental data / H. S. Ahluwalia, M. M. Fikani // Journal of Geophysical Research. - 1996. - V. 101. - No. A5. P. 11075-11085. 